\title{
Random Walks on Digraphs, the Generalized Digraph Laplacian and the Degree of Asymmetry
}

\author{
Yanhua Li and Zhi-Li Zhang* \\ University of Minnesota, Twin Cities \\ \{yanhua,zhzhang\}@cs.umn.edu
}

\begin{abstract}
In this paper we extend and generalize the standard random walk theory (or spectral graph theory) on undirected graphs to digraphs. In particular, we introduce and define a (normalized) digraph Laplacian matrix, and prove that 1) its Moore-Penrose pseudo-inverse is the (discrete) Green's function of the digraph Laplacian matrix (as an operator on digraphs), and 2) it is the normalized fundamental matrix of the Markov chain governing random walks on digraphs. Using these results, we derive new formula for computing hitting and commute times in terms of the Moore-Penrose pseudo-inverse of the digraph Laplacian, or equivalently, the singular values and vectors of the digraph Laplacian. Furthermore, we show that the Cheeger constant defined in [6] is intrinsically a quantity associated with undirected graphs. This motivates us to introduce a metric - the largest singular value of $\Delta:=\left(\tilde{\mathcal{L}}-\tilde{\mathcal{L}}^{T}\right) / 2$ - to quantify and measure the $d e-$ gree of asymmetry in a digraph. Using this measure, we establish several new results, such as a tighter bound (than that of Fill's in [9] and Chung's in [6]) on the Markov chain mixing rate, and a bound on the second smallest singular value of $\tilde{\mathcal{L}}$.
\end{abstract}

\section{Introduction}

Graphs arising from many applications such as web are directed, where direction of links contains crucial information. Random walks are frequently used to model certain dynamic processes on (directed or undirected) graphs, for example, to reveal important network structural information, e.g., importance of nodes as in the Page-Rank algorithm [5], or to study ways to efficiently explore complex networks.

Random walks on undirected graphs have been extensively studied and are wellunderstood (see [13]). They are closely related to spectral graph theory [7], which has produced powerful tools to study many important properties of (undirected) graphs that are of both theoretical and practical significance. Well-known results include bounds on Cheeger constant and mixing rate in terms of the second smallest eigenvalue of the graph Laplacian. On the other hand, there are relatively few similar studies on directed graphs, see, e.g., $[6,15]$, where the authors circumvent the "directedness" of digraphs by converting them into undirected graphs through symmetrization.

\footnotetext{
* The work was supported in part by the National Science Foundation grants CNS-0905037 and CNS-1017647, the DTRA grant HDTRA1-09-1-0050, and a University of Minnesota DTC DTI grant.
} 
In this paper we develop a spectral graph theory (or random walk theory) for $d i$ rected graphs (in short, digraphs). We introduce the notion of digraph Laplacian, a direct generalization of the graph Laplacian (for undirected graphs), denoted by $\tilde{\mathcal{L}}$. Instead of using the node degrees (as in the case of undirected graphs), the digraph Laplacian is defined using stationary probabilities of the Markov chain governing random walks on digraphs. Furthermore, instead of relying on the positive semi-definiteness of the graph Laplacian matrix (for undirected graphs), we establish a key connection between the digraph Laplacian $\tilde{\mathcal{L}}$ and its Moore-Penrose pseudo-inverse [10], denoted by $\tilde{\mathcal{L}}^{+}$, and use the properties of this connection to prove several parallel results for random walks on digraphs. In particular, we show that 1) the Moore-Penrose pseudo-inverse $\tilde{\mathcal{L}}^{+}$of the digraph Laplacian is exactly the (discrete) Green's function of the digraph Laplacian matrix $\tilde{\mathcal{L}}$, acting as an operator on digraphs [8], and 2) $\tilde{\mathcal{L}}^{+}$is the normalized fundamental matrix [1] of the Markov chain governing random walks on digraphs.

Based on the connection between $\tilde{\mathcal{L}}^{+}$and the fundamental matrix, we show how hitting and commute times can be directly computed in terms of the singular values and vectors of the digraph Laplacian - this yields a more direct and efficient way to compute hitting and commute times than existing methods based on the fundamental matrix. More generally, our results suggest a "spectral graph theory" for digraphs, where graph properties can be studied using the singular values of the digraph Laplacian in place of the eigenvalues of the graph Laplacian. In particular, our theory of random walks on digraphs subsumes the existing theory of random walks on undirected graphs as a special case.

Furthermore, we show that the well-known Cheeger constant-generalized by Chung to digraphs in [6] - is fundamentally a quantity intrinsic to undirected graphs, as there are infinitely many digraphs with the same symmetrized (undirected) graph. Hence bounds based on the eigenvalues of the symmetrized graph Laplacian do not uniquely capture the properties of digraphs. This leads us to introduce the degree of asymmetry to capture the overall "directedness" of digraphs, formally defined as follows: we express a digraph Laplacian $\tilde{\mathcal{L}}$ in terms of a symmetric part, $\overline{\mathcal{L}}=\left(\tilde{\mathcal{L}}+\tilde{\mathcal{L}}^{T}\right) / 2$, and a skew-symmetric part, $\Delta=\left(\tilde{\mathcal{L}}-\tilde{\mathcal{L}}^{T}\right) / 2$. ( $(\overline{\mathcal{L}}$ is the (symmetrized) graph Laplacian for digraphs introduced by Chung in [6].) The largest singular value of $\Delta, \delta_{\max }$, is referred to as the degree of asymmetry, which provides a quantitative measure of the asymmetry in digraphs. Many key properties of digraphs can then be bounded by the eigenvalues of $\overline{\mathcal{L}}$ and the degree of asymmetry. For instance, by accounting for the asymmetry of digraphs, we are able to obtain a tighter bound (than that of Fill's in [9] and Chung's in [6]) on (non-reversible) Markov chain mixing rate.

\section{Preliminaries: Random Walks on Undirected Graphs}

We use a triple $G=(V, E, A)$ to denote an undirected and weighted graph on the node set $V=\{1,2, \ldots, n\}$. The $n \times n$ (nonnegative) weight matrix $A=\left[a_{i j}\right]$ is symmetric, and is defined in such a way that $a_{i j}=a_{j i}>0$, if $\langle i, j\rangle \in E$, and $a_{i j}=a_{j i}=0$ otherwise. For $1 \leq i \leq n$, the degree of node $i$ is $d_{i}=\sum_{j=1}^{n} a_{i j}$. The volume of $G$, denoted by $\operatorname{vol}(G)$, is defined as the sum of all node degrees, $d=\sum_{i=1}^{n} d_{i}$, i.e., $\operatorname{vol}(G)=d$. 
A random walk on $G$ is a Markov chain defined on $G$ with the transition probability matrix $P=\left[p_{i j}\right]$, where $p_{i j}=a_{i j} / d_{i}$. Let $D=\operatorname{diag}\left[d_{i}\right]$ be a diagonal matrix of node degrees, then $P=D^{-1} A$. Without loss of generality, we assume that the undirected graph $G$ is connected (i.e., any node can reach any other node in $G$ ). Then it can be shown (see, e.g., [1]) that the Markov chain is irreducible, and there exists a unique stationary distribution, $\left\{\pi_{1}, \pi_{2}, \ldots, \pi_{n}\right\}$. Let $\pi=\left[\pi_{i}\right]_{1 \leq i \leq n}$ be the column vector of the stationary probabilities. Then $\pi^{T} P=\pi^{T}$, where the superscript $T$ represents (vector or matrix) transpose. Furthermore, this Markov chain (random walk) on $G$ is reversible, namely

$$
\pi_{i} p_{i j}=\pi_{j} p_{j i}, \text { for any } i, j,
$$

and

$$
\pi_{i}=\frac{d_{i}}{\sum_{k} d_{k}}=\frac{d_{i}}{d}, i=1,2, \ldots, n .
$$

Following [7], we will use the normalized graph Laplacian (instead of the unnormalized version $L=D-A$ ). Given an undirected $G$, the normalized graph Laplacian of $G$ (also called normalized Laplacian matrix of $G$ ) is defined as follows:

$$
\mathcal{L}=D^{-\frac{1}{2}}(D-A) D^{-\frac{1}{2}}=D^{\frac{1}{2}}(I-P) D^{-\frac{1}{2}}
$$

A key property of the graph Laplacian (for an undirected graph) is that $\mathcal{L}$ is symmetric and positive semi-definite [10]. Hence all eigenvalues of $\mathcal{L}$ are nonnegative real numbers. In particular, for a connected undirected graph $G, \mathcal{L}$ has rank $n-1$ and has exactly one zero eigenvalue (its smallest one). Let $\lambda_{1}=0<\lambda_{2} \leq \cdots \leq \lambda_{n}$ be the $n$ eigenvalues of $\mathcal{L}$ arranged in an increasing order, and $\mu_{i}, 1 \leq i \leq n$, be the corresponding eigenvectors (of unit norm). In particular, one can show that the (column) eigenvector, $\mu_{1}$, of $\mathcal{L}$ associated with the eigenvalue $\lambda_{1}=0$, is given by

$$
\mu_{1}=\pi^{\frac{1}{2}}=\left[\sqrt{\pi_{i}}\right]=\left[\frac{\sqrt{d_{i}}}{\sqrt{d}}\right] .
$$

Define $\Gamma:=\operatorname{diag}\left[\lambda_{1}, \ldots, \lambda_{n}\right]$, the diagonal matrix formed by the eigenvalues, and $U=\left[\mu_{1}, \ldots, \mu_{n}\right]$, an orthonormal matrix formed by the eigenvectors of $\mathcal{L}$, where $U U^{T}=U^{T} U=I$. It is easy to see that the graph Laplacian $\mathcal{L}$ admits an eigendecomposition [10], namely, $\mathcal{L}=U \Gamma U^{T}$. Using the eigenvalues and eigenvectors of $\mathcal{L}$, we can compute the hitting times and commute times using the following formula [13]:

$$
H_{i j}=\sum_{k>1} \frac{d}{\lambda_{k}}\left(\frac{\mu_{k j}^{2}}{d_{j}}-\frac{\mu_{k i} \mu_{k j}}{\sqrt{d_{i} d_{j}}}\right),
$$

and

$$
C_{i j}=\sum_{k>1} \frac{d}{\lambda_{k}}\left(\frac{\mu_{k i}}{\sqrt{d_{i}}}-\frac{\mu_{k j}}{\sqrt{d_{j}}}\right)^{2},
$$

where $\mu_{k j}$ is the $j$ th entry of the column vector $\mu_{k}$. 


\section{Random Walk Theory on Digraphs}

In this section, we develop the random walk theory for digraphs. In particular, we generalize the graph Laplacian defined for undirected graphs, and introduce the digraph Laplacian matrix. We prove that the Moore-Penrose pseudo-inverse of this digraph Laplacian is exactly equal to (a normalized version of) the fundamental matrix of the Markov chain governing random walks on digraphs, and show that it is also the Green's function of the digraph Laplacian. Using these connections, we illustrate that how hitting and commute times of random walks on digraphs can be directly computed using the singular values and vectors of the digraph Laplacian. We also show that when the underlying graph is undirected, our results reduce to the well-known results for undirected graphs. Hence our theory includes undirected graphs as a special case.

\subsection{Random Walks on Directed Graphs and Fundamental Matrix}

As alluded earlier, random walks can be defined not only on undirected graphs, but also on digraphs. Let $G=(V, E, A)$ be a (weighted) digraph defined on the vertex set $V=\{1,2, \ldots, n\}$, where $A$ is a nonnegative, but generally asymmetric weight matrix such that $a_{i j}>0$ if and only if the directed edge (or arc) $\langle i, j\rangle \in E$. As before, we will simply refer to $A$ as the adjacency matrix of $G$. For $i=1,2, \ldots, n$, we define the outdegree of vertex $i, d_{i}^{+}=\sum_{j=1}^{n} a_{i j}$, and the in-degree of vertex $i, d_{i}^{-}=\sum_{j=1}^{n} a_{j i}$. In general, $d^{+} \neq d^{-}$. However, we have $d:=\sum_{i=1}^{n} d_{i}^{+}=\sum_{i=1}^{n} d_{i}^{-}=\sum_{i=1}^{n} \sum_{j=1}^{n} a_{i j}$. As before, we refer to $d$ as the volume of the directed graph $G$, i.e., $\operatorname{vol}(G)=d$. For conciseness, in the following unless otherwise stated, we refer to the out-degree of a vertex simply as its degree, and use $d_{i}$ for $d_{i}^{+}$.

Let $D=\operatorname{diag}\left[d_{i}\right]$ be a diagonal matrix of the vertex out-degrees, and define $P=$ $D^{-1} A$. Then $P=\left[p_{i j}\right]$ is the transition probability matrix of the Markov chain associated with random walks on $G$, where at each vertex $i$, a random walk has the probability $p_{i j}=a_{i j} / d_{i}$ to transit from vertex $i$ to vertex $j$, if $\langle i, j\rangle \in E$. We assume that $G$ is strongly connected, i.e., there is a (directed) path from any vertex $i$ to any other vertex $j$. Then the Markov chain $P$ is irreducible, and has a unique stationary probability distribution, $\left\{\pi_{i}\right\}$, where $\pi_{i}>0,1 \leq i \leq n$. Namely, $\pi^{T} P=\pi^{T}$, where $\pi=\left[\pi_{1}, \ldots, \pi_{n}\right]^{T}$ be the (column) vector of stationary probabilities. Unlike undirected graphs, the Markov chain associated with random walks on directed graphs is generally non-reversible, and eqs.(1) and (2) for undirected graphs do not hold.

For random walks on directed graphs, quantities such as hitting times and commute times can be defined exactly as in the case of undirected graphs. However, since the (normalized) Laplacian matrix $\mathcal{L}$ is (so far!) defined only for undirected graphs, we cannot use the relations eqs.(5) and (6) to compute hitting times and commute times for random graphs on directed graphs. On the other hand, using results from the standard Markov chain theory, we can express the hitting times and commute times in terms of the fundamental matrix. In [1], Aldous and Fill define the fundamental matrix $Z=\left[z_{i j}\right]$ for an irreducible Markov chain with the transition probability matrix $P$ :

$$
z_{i j}=\sum_{t=0}^{\infty}\left(p_{i j}^{(t)}-\pi_{j}\right), 1 \leq i, j \leq n,
$$


where $p_{i j}^{(t)}$ is the $(i, j)$-th entry in the $t$-step transition probability matrix $P^{t}=\underbrace{P \cdots P}_{t}$.

Let $\Pi=\operatorname{diag}\left[\pi_{i}\right]$ be the diagonal matrix containing the stationary probabilities $\pi_{i}$ 's on the diagonal, and $J=\left[J_{i j}\right]$ the all-one matrix, i.e., $J_{i j}=1,1 \leq i, j \leq n$. We can express $Z$ alternatively as the sum of an infinite matrix series:

$$
Z=\sum_{t=0}^{\infty}\left(P^{t}-J \Pi\right)=\sum_{t=0}^{\infty}\left(P^{t}-\mathbf{1} \pi^{T}\right)
$$

where $\mathbf{1}=[1, \ldots, 1]^{T}$ is the all-one column vector. Hence $J=\mathbf{1} \cdot \mathbf{1}^{T}$, and $\mathbf{1}^{T} \Pi=\pi^{T}$.

While the physical meaning of the fundamental matrix $Z$ may not be obvious from its definition eq.(7) (or eq.(8)), it plays a crucial role in computing various quantities related to random walks, or more generally, various stopping time properties of Markov chains [1]. For instance, the hitting times and commute times of random walks on a directed graph can be expressed in terms of $Z$ as follows (see [1]):

$$
H_{i j}=\frac{z_{j j}-z_{i j}}{\pi_{j}}
$$

and

$$
C_{i j}=\frac{z_{j j}-z_{i j}}{\pi_{j}}+\frac{z_{i i}-z_{j i}}{\pi_{i}} .
$$

In eqs.(7) and (8), the fundamental matrix $Z$ is defined as an infinite sum. We show that $Z$ in fact satisfies a simple relation eq.(11), and hence can be computed directly using the standard matrix inverse.

Theorem 1. Let $P$ be the transition probability matrix for an irreducible Markov chain. Then its corresponding fundamental matrix $Z$ as defined in eq.(7) satisfies the following relation

$$
Z+J \Pi=(I-P+J \Pi)^{-1}
$$

Proof: Note that $J \Pi=\mathbf{1} \pi^{T}$. From $\pi^{T} P=\pi^{T}$ and $P \mathbf{1}=\mathbf{1}$, we have $J \Pi P=J \Pi$ and $P J \Pi=J \Pi$. Using these two relations, it is easy to prove the following equation by induction.

$$
P^{m}-J \Pi=(P-J \Pi)^{m}, \text { for any integer } m>0 \text {. }
$$

Plugging this into eq.(8) yields Theorem 1.

As undirected graphs are a special case of directed graphs, eqs.(9) and (10) provide an alternative way to compute hitting times and commute times for random walks on fully connected undirected graphs. In this paper we will show that eqs.(5) and (6) are in fact equivalent to eqs.(9) and (10). 


\section{2 (Normalized) Digraph Laplacian and Green's Function for Digraphs}

We now generalize the existing spectral graph theory defined for undirected graphs to directed graphs by introducing an appropriately generalized Laplacian matrix for (strongly connected) diagraphs. Let $G=(V, E, A)$ be a strongly connected (weighted) digraph defined on the vertex set $V=\{1,2, \ldots, n\}$, where in general the weight (or adjacency) matrix $A$ is asymmetric. A major technical difficulty in dealing with digraphs is that if one naively extends the (normalized) Laplacian matrix, $\mathcal{L}=D^{-1 / 2}(D-$ A) $D^{-1 / 2}$, (or its un-normalized version, $L=D-A$ ), defined for undirected graphs to digraphs, $\mathcal{L}$ is in general asymmetric; hence the nice properties such as positive semidefiniteness of $\mathcal{L}$ no longer hold. Past attempts in generalizing the spectral graph theory to digraphs have been simply symmetrized $\mathcal{L}$, e.g., by introducing a symmetric matrix, $\overline{\mathcal{L}}:=\left(\mathcal{L}+\mathcal{L}^{T}\right) / 2[6,15]$. Unfortunately, as will be shown in the Section 4 , such symmetrized $\overline{\mathcal{L}}$ does not directly capture the unique characteristic of the random walk on the digraph as defined earlier, since a set of diagraphs can have the same $\overline{\mathcal{L}}$.

For a strongly connected digraph $G$, let $\Pi^{\frac{1}{2}}=\operatorname{diag}\left[\sqrt{\pi_{i}}\right]$. We define the (normalized) digraph Laplacian for $G$ (also referred to as the generalized (normalized) Laplacian matrix $\left.{ }^{1}\right), \tilde{\mathcal{L}}=\left[\tilde{\mathcal{L}}_{i j}\right]$ as follows:

\section{Definition 1 (Normalized Digraph Laplacian $\tilde{\mathcal{L}}$ ).}

$$
\tilde{\mathcal{L}}=\Pi^{\frac{1}{2}}(I-P) \Pi^{-\frac{1}{2}},
$$

namely, for $1 \leq i, j \leq n$,

$$
\tilde{\mathcal{L}}_{i j}= \begin{cases}1-p_{i i} & \text { if } i=j, \\ -\pi_{i}^{\frac{1}{2}} p_{i j} \pi_{j}^{-\frac{1}{2}} & \text { if }\langle i, j\rangle \in E, \\ 0 & \text { otherwise. }\end{cases}
$$

Treating this (normalized) digraph Laplacian matrix $\tilde{\mathcal{L}}$ as an (asymmetric) operator on a digraph $G$, we now define the (discrete) Green's function $\tilde{\mathcal{G}}$ (without boundary conditions) for digraphs in exactly the same manner as for undirected graphs [8]. Namely, $\tilde{\mathcal{G}}$ is a matrix with its entries, indexed by vertices $i$ and $j$, that satisfies the following conditions:

$$
[\tilde{\mathcal{G}} \tilde{\mathcal{L}}]_{i, j}=I_{i, j}-\sqrt{\pi_{i} \pi_{j}}, 1 \leq i, j \leq n,
$$

and expressed in the matrix form,

$$
\tilde{\mathcal{G}} \tilde{\mathcal{L}}=I-\pi^{\frac{1}{2}} \pi^{\frac{1}{2}}{ }^{T} .
$$

In the following we will show that $\tilde{\mathcal{G}}$ is precisely $\tilde{\mathcal{L}}^{+}$, the pseudo-inverse of the Laplacian operator $\tilde{\mathcal{L}}$ on the digraph $G$. Furthermore, we will relate $\tilde{\mathcal{L}}^{+}$directly to the fundamental matrix $Z$ of the Markov chain associated with random walks on the digraph $G$. Before we establish a main result of this paper, we first introduce a few more notations and then prove the following useful lemma.

\footnotetext{
${ }^{1}$ An un-normalized digraph Laplacian is defined as $L=\Pi(I-P)$ in [3].
} 
Lemma 1. Define $\tilde{\mathcal{Z}}=\Pi^{\frac{1}{2}} Z \Pi^{-\frac{1}{2}}$ (the normalized fundamental matrix), and $\tilde{J}=$ $\Pi^{\frac{1}{2}} J \Pi^{\frac{1}{2}}=\pi^{\frac{1}{2}} \pi^{\frac{1}{2}}{ }^{T}$. The following relations regarding $\tilde{\mathcal{Z}}$ and $\tilde{J}$ hold: (1) $\tilde{J}=\tilde{J}^{2}$, (2) $\tilde{J} \tilde{\mathcal{L}}=\tilde{\mathcal{L}} \tilde{J}=\tilde{\mathcal{L}} \pi^{\frac{1}{2}}=\pi^{\frac{1}{2}}{ }^{T} \tilde{\mathcal{L}}=0$, and (3) $\tilde{J} \tilde{\mathcal{Z}}=\tilde{\mathcal{Z}} \tilde{J}=\tilde{\mathcal{Z}} \pi^{\frac{1}{2}}=\pi^{\frac{1}{2}} \tilde{\mathcal{Z}}=0$.

Proof Sketch: These relations can be established using the facts that $J=\mathbf{1 1}^{T}, \mathbf{1}^{T} \Pi=$ $\pi^{T}, \pi^{T} J=\mathbf{1}^{T}, \Pi J=\pi \mathbf{1}^{T}, J \Pi J=J, \pi^{T}(I-P)=0,(I-P) \mathbf{1}=0, \pi^{T} Z=0$, and $Z \mathbf{1}=0$. The last four equalities imply that the matrices $I-P$ and $Z$ have the same left and right eigenvectors, $\pi^{T}$ and $\mathbf{1}$, corresponding to the eigenvalue 0 .

We are now in a position to prove a main theorem of the paper, which states the Green's function for the (normalized) digraph Laplacian is exactly its Moore-Penrose pseudo-inverse, and it is equal to the normalized fundamental matrix. Namely, $\tilde{\mathcal{G}}=$ $\tilde{\mathcal{L}}^{+}=\tilde{\mathcal{Z}}$. For completeness, we also include the key definitions in the statement of the theorem.

Theorem 2 (Laplacian matrix and Green's function for digraphs). Given a strongly connected digraph $G=(V, E, A)$ where $V=\{1, \ldots, n\}$ and $A$ is a (generally asymmetric) nonnegative weight/adjancency matrix of $G$ such that $a_{i j}>0$ if and only if $\langle i, j\rangle \in E$, let $D=\operatorname{diag}\left[d_{i}\right]$ be the diagonal (out-)degree matrix, i.e., $d_{i}=\sum_{j} a_{i j}$. Then $P=D^{-1} A$ is the transition probability matrix for the (irreducible and generally non-reversible) Markov chain associated with random walks on the digraph $G$. Let $\pi=\left[\pi_{1}, \ldots, \pi_{n}\right]^{T}$ be the stationary probability distribution (represented as a column vector) for the Markov chain $P$, and $\Pi=\operatorname{diag}\left[\pi_{i}\right]$ be the diagonal stationary probability matrix. We define the (normalized) digraph Laplacian matrix $\tilde{\mathcal{L}}$ of $G$ as in eq.(13), i.e., $\tilde{\mathcal{L}}=\Pi^{\frac{1}{2}}(I-P) \Pi^{-\frac{1}{2}}$. Define $\tilde{\mathcal{Z}}=\Pi^{\frac{1}{2}} Z \Pi^{-\frac{1}{2}}$, where $Z$ is the fundamental matrix of the Markov chain $P$ as defined in eq.(7).

Then $\tilde{\mathcal{Z}}=\tilde{\mathcal{L}}^{+}$, is the pseudo-inverse of the Laplacian matrix $\tilde{\mathcal{L}}$. Furthermore, $\tilde{\mathcal{Z}}$ is the (discrete) Green's function for $\tilde{\mathcal{L}}$. Namely,

$$
\tilde{\mathcal{Z}} \tilde{\mathcal{L}}=I-\Pi^{\frac{1}{2}} J \Pi^{\frac{1}{2}}=I-\pi^{\frac{1}{2}} \pi^{\frac{1}{2}}
$$

where $J$ is the all-one matrix and $\pi^{\frac{1}{2}}=\left[\pi_{1}^{\frac{1}{2}}, \ldots, \pi_{n}^{\frac{1}{2}}\right]^{T}$ (a column vector).

Proof Sketch: From eq.(11) in Theorem 1, we have

$$
\tilde{\mathcal{Z}}+\tilde{J}=(\tilde{\mathcal{L}}+\tilde{J})^{-1} .
$$

Multiplying eq.(18) from the right by $\tilde{\mathcal{L}}+\tilde{J}$, and using Lemma 1 , it is easy to see that

$$
\tilde{\mathcal{Z}} \tilde{\mathcal{L}}=I-\tilde{J}
$$

which establishes that $\tilde{\mathcal{Z}}$ is the Green's function of the digraph Laplacian $\tilde{\mathcal{L}}$. Similarly, by multiplying eq.(18) from the left by $\tilde{\mathcal{L}}+\tilde{J}$, we can likewise prove $\tilde{\mathcal{L}} \tilde{\mathcal{Z}}=I-\tilde{J}$. Hence $\tilde{\mathcal{Z}} \tilde{\mathcal{L}}=\tilde{\mathcal{L}} \tilde{\mathcal{Z}}=I-\tilde{J}$, which is a real symmetric matrix. Hence $(\tilde{\mathcal{L}} \tilde{\mathcal{Z}})^{T}=\tilde{\mathcal{L}} \tilde{\mathcal{Z}}$ and $(\tilde{\mathcal{Z}} \tilde{\mathcal{L}})^{T}=\tilde{\mathcal{Z}} \tilde{\mathcal{L}}$. Furthermore, as $\tilde{J} \tilde{\mathcal{Z}}=0$, eq.(19) yields $\tilde{\mathcal{Z}} \tilde{\mathcal{L}} \tilde{\mathcal{Z}}=\tilde{\mathcal{Z}}$. Similarly, as $\tilde{\mathcal{L}} \tilde{J}=$ $\Pi^{\frac{1}{2}}(I-P) J \Pi^{\frac{1}{2}}=0$, eq.(19) yields $\tilde{\mathcal{L}} \tilde{\mathcal{Z}} \tilde{\mathcal{L}}=\tilde{\mathcal{L}}$. These establish that $\tilde{\mathcal{Z}}$ satisfies the four conditions of matrix pseudo-inverse. Hence $\tilde{\mathcal{Z}}$ is also the Moore-Penrose pseudoinverse of $\tilde{\mathcal{L}}$. Therefore, $\tilde{\mathcal{G}}=\tilde{\mathcal{Z}}=\tilde{\mathcal{L}}^{+}$. 


\subsection{Computing Hitting and Commute Times for Digraphs using Digraph Laplacian}

Using the relationship between the (normalized) digraph Laplacian $\tilde{\mathcal{L}}$, its pseudo-inverse $\tilde{\mathcal{L}}^{+}$, and the (normalized) fundamental matrix $\tilde{\mathcal{Z}}$, we can now express the hitting times and commute times of random walks on digraphs in terms of $\hat{\mathcal{L}}^{+}$, or alternatively in terms of the singular values and singular vectors of the digraph Laplacian matrix $\tilde{\mathcal{L}}$.

From $Z=\Pi^{-\frac{1}{2}} \tilde{\mathcal{Z}} \Pi^{\frac{1}{2}}=\Pi^{-\frac{1}{2}} \tilde{\mathcal{L}}^{+} \Pi^{\frac{1}{2}}$, and using eqs.(9) and (10), we can compute the hitting times and commute times for random walks on digraphs directly in terms of the entries of $\tilde{\mathcal{L}}^{+}$.

Theorem 3 (Computing hitting and commute times using $\tilde{\mathcal{L}}^{+}$). The hitting times and commute times of random walks on a strongly connected digraphs can be computed using the pseudo-inverse of the (generalized) normalized Laplacian matrix $\tilde{\mathcal{L}}^{+}$ as follows:

$$
H_{i j}=\frac{{\tilde{\mathcal{L}_{j j}}}^{+}}{\pi_{j}}-\frac{\tilde{\mathcal{L}_{i j}}{ }^{+}}{\sqrt{\pi_{i} \pi_{j}}},
$$

and

$$
C_{i j}=H_{i j}+H_{j i}=\frac{{\tilde{\mathcal{L}_{j j}}}^{+}}{\pi_{j}}+\frac{{\tilde{\mathcal{L}_{i i}}}^{+}}{\pi_{i}}-\frac{{\tilde{\mathcal{L}_{i j}}}^{+}}{\sqrt{\pi_{i} \pi_{j}}}-\frac{{\tilde{\mathcal{L}_{j i}}}^{+}}{\sqrt{\pi_{i} \pi_{j}}}
$$

where ${\tilde{\mathcal{L}_{i j}}}^{+}$is the $(i, j)$-th entry of $\tilde{\mathcal{L}}^{+}$, and $\pi_{i}$ is the stationary probability of vertex $i$.

For undirected graphs, we show that eqs.(20) and (21) reduce to eqs.(5) and (6) in Section 2 . This can be seen from the fact that for undirected graphs, $\tilde{\mathcal{L}}=\mathcal{L}$ is symmetric and positive semi-definite. Hence the singular value decomposition of $\mathcal{L}$ is the same as the eigen-decomposition of $\mathcal{L}$.

\section{Degree of Asymmetry, Generalized Cheeger Constant and Bounds on Mixing Rate}

In this section we explore the relation between digraph Laplacian $\tilde{\mathcal{L}}$ and its symmetrized version $\overline{\mathcal{L}}$. We first show that the symmetrized Laplacian matrix $\overline{\mathcal{L}}$, and the Cheeger constant $h(G)$ as defined in [6] are in a sense primarily determined by an undirected graph associated with the random walks with the transition probability matrix $\bar{P}=$ $\left(P+\Pi^{-1} P^{T} \Pi\right) / 2$, thus cannot capture the unique characteristics of each individual diagraph. As a result, we investigate two questions: 1) how can the "degree of asymmetry" of a digraph be quantified and measured? and 2) how does the degree of asymmetry affect crucial properties of a digraph such as the mixing rate? In the following we propose one metric - the largest singular value of $\Delta:=\left(\tilde{\mathcal{L}}-\tilde{\mathcal{L}}^{T}\right) / 2-$ as a measure of the degree of asymmetry in a digraph. We show that by explicitly accounting for the degree of asymmetry, we can obtain generally tighter bounds on quantities (e.g., mixing rate) associated with random walks (or Markov chains) on digraphs. 


\subsection{The Degree of Asymmetry, and Relations to Symmetrized Digraph Laplacian}

In [6], Chung introduces the symmetrized Laplacian matrix for digraphs, $\overline{\mathcal{L}}=\frac{\tilde{\mathcal{L}}+\tilde{\mathcal{L}}^{T}}{2}$, generalizes the Cheeger constant to digraphs and bounds it in terms of the second smallest eigenvalue of $\overline{\mathcal{L}}$. In the following we show that the symmetrized Laplacian $\overline{\mathcal{L}}$ and the Cheeger constant introduced by Chung are in fact two quantities intrinsic to undirected graphs.

Theorem 4. Given a digraph $G$, with transition probability matrix $P$, there exist infinite digraphs which have the same stationary distribution matrix $\Pi$ and the same symmetrized transition probability matrix $\bar{P}=\left(P+\Pi^{-1} P^{T} \Pi\right) / 2$. As a result, all these graphs have the same symmetrized Laplacian matrix and Cheeger constant.

Proof : We prove it by construction. Given a digraph $G=(V, E, A)$, with transition probability matrix $P$, all the digraphs $G^{\prime}$ 's with the transition probability $P^{\prime}$ as

$$
P^{\prime}(\alpha)=\alpha P+(1-\alpha) \Pi^{-1} P^{T} \Pi,
$$

form an infinite digraph set, denoted by $\mathcal{G}(G)$, where $\alpha \in[0,1]$.

(1)It is easy to check that any $P^{\prime}(\alpha)$ defined in eq.(22) is non-negative, and satisfies $\pi^{T} P^{\prime}(\alpha)=\pi^{T}$, and $P^{\prime}(\alpha) \mathbf{1}=\mathbf{1}$, thus $P^{\prime}(\alpha)$ represents a transition probability matrix of a random walk with stationary distribution $\pi$.

For any $G^{\prime} \in \mathcal{G}(G)$, the digraph Laplacian matrix is given by $\tilde{\mathcal{L}}^{\prime}=\Pi^{\frac{1}{2}}(I-$ $\left.P^{\prime}\right) \Pi^{-\frac{1}{2}}$, and the symmetrized Laplacian is only determined by $\bar{P}$, since we have

$$
\overline{\mathcal{L}}=\frac{\tilde{\mathcal{L}}^{\prime}+\tilde{\mathcal{L}}^{\prime T}}{2}=\Pi^{\frac{1}{2}}\left(I-\frac{P+\Pi^{-1} P^{T} \Pi}{2}\right) \Pi^{-\frac{1}{2}}=\Pi^{\frac{1}{2}}(I-\bar{P}) \Pi^{-\frac{1}{2}} .
$$

(2) In particular, when $\alpha=\frac{1}{2}, P^{\prime}\left(\frac{1}{2}\right)=\bar{P}$ represents the undirected graph $\bar{G}$. For any $S \subset N:=\{1, \ldots, n\}$, define an $n$-element vector $f_{S}$, where $f_{S}(i)=\frac{1}{F_{\pi}(S)}, i \in S$ and $f_{S}(i)=-\frac{1}{F_{\pi}(S)}, i \in \bar{S}$, where $F_{\pi}(S):=\sum_{i \in S} \pi_{i}$ is the circulation function [6]. Define $x_{S}=\Pi^{-\frac{1}{2}} f_{S}$. Then

$$
\min _{S} \frac{x_{S}^{T} \tilde{\mathcal{L}} x_{S}}{x_{S}^{T} x_{S}}=\min _{S} \frac{f_{S}^{T} \Pi(I-P) f_{S}}{f_{S}^{T} \Pi f_{S}} \leq 2 \inf _{S} \frac{F_{\pi}(\partial S)}{\min \left\{F_{\pi}(S), F_{\pi}(\bar{S})\right\}}=2 h(G) .
$$

The above inequality indicates that the Cheeger constant $h(G)$ is closely related to $\min _{S} \frac{x_{S}^{T} \tilde{\mathcal{L}} x_{S}}{x_{S}^{T} x_{S}}$. On the other hand, $x_{S}^{T} \tilde{\mathcal{L}} x_{S}=x_{S}^{T} \tilde{\mathcal{L}}^{T} x_{S}=\frac{1}{2}\left(x_{S}^{T} \tilde{\mathcal{L}} x_{S}+x_{S}^{T} \tilde{\mathcal{L}}^{T} x_{S}\right)=$ $x_{S}^{T} \overline{\mathcal{L}} x_{S}$. Hence for any digraph $G^{\prime}$ with a digraph Laplacian $\tilde{\mathcal{L}}^{\prime}$ such that $\left(\tilde{\mathcal{L}}^{\prime}+\tilde{\mathcal{L}}^{\prime}{ }^{T}\right) / 2=$ $\overrightarrow{\mathcal{L}}$, we have $x_{S}^{T} \tilde{\mathcal{L}}^{\prime} x_{S}=x_{S}^{T} \overline{\mathcal{L}} x_{S}$. We see that the left-hand side of eq.(24) hinges only on $\overline{\mathcal{L}}$. Therefore, any graph $G^{\prime} \in \mathcal{G}(G)$ has the same Cheeger constant, i.e. $h\left(G^{\prime}\right)=h(G)=h(\bar{G})$.

To capture the "degree of asymmetry" in a digraph, we express $\tilde{\mathcal{L}}$ as a sum of a symmetric part and a skew-symmetric part: $\tilde{\mathcal{L}}=\overline{\mathcal{L}}+\Delta$, where $\Delta=\left(\tilde{\mathcal{L}}-\tilde{\mathcal{L}}^{T}\right) / 2$. Note that $\tilde{\mathcal{L}}^{T}=\overline{\mathcal{L}}+\Delta^{T}=\overline{\mathcal{L}}-\Delta$. Hence $\Delta$ captures the difference between $\tilde{\mathcal{L}}$ and 
its transpose (which induces a reserved Markov chain or random walk). When $\tilde{\mathcal{L}}$ is symmetric, then $\Delta=0$. Let $(0=) \sigma_{1} \leq \sigma_{2} \leq \ldots \leq \sigma_{n}$ denote the singular values (in an increasing order) of $\tilde{\mathcal{L}}$. Likewise, let $(0=) \bar{\lambda}_{1} \leq \bar{\lambda}_{2} \leq \ldots \leq \bar{\lambda}_{n}$ denote the eigenvalues of $\overline{\mathcal{L}}$, and $(0=) \delta_{1} \leq \delta_{2} \leq \ldots \leq \delta_{n}\left(=\delta_{\max }\right)$ the singular values of $\Delta$. The following relations among them hold (See [2]):

$$
\bar{\lambda}_{i} \leq \sigma_{i} \leq \bar{\lambda}_{i}+\delta_{n}, i=1,2, \ldots, n .
$$

From eq.(25), we see that $\sigma_{i}-\bar{\lambda}_{i} \leq \delta_{n}, i=2, \ldots, n$. We therefore propose the largest singular value of $\Delta, \delta_{n}\left(=\delta_{\max }\right)$ as a measure of the degree of asymmetry in the underlying digraph. Note that $\delta_{n}=\|\Delta\|$, where $\|\cdot\|$ is the operator (bound) norm of a matrix: $\|\Delta\|:=\sup _{\|x\|=1}\|\Delta x\|_{2}=\sup _{\|y\|=\|x\|=1}\left|\langle y, \tilde{\mathcal{L}} x\rangle-\left\langle y, \tilde{\mathcal{L}}^{T} x\right\rangle\right|=$ $\sup _{\|y\|=\|x\|=1}|\langle y, \tilde{\mathcal{L}} x\rangle-\langle x, \tilde{\mathcal{L}} y\rangle|$ (see, e.g., [2], p.6 and p.91). On the other hand, $\langle x, \tilde{\mathcal{L}} x\rangle-\left\langle x, \tilde{\mathcal{L}}^{T} x\right\rangle=0$ for any $x$. In the following, we relate and bound $\delta_{n}-$ the degree of asymmetry - to two other important quantities associated with the Markov chain on a digraph: the digraph gap $g(G)$ defined below and the second largest singular value of the transmission probability matrix $P$.

Given a digraph $G$, the circulation function $F_{\pi}(\cdot)$, where $F_{\pi}(i, j)=\pi_{i} P_{i j}$, obeys the flow conservation law at every node of a digraph: $\sum_{k} F(k, i)=\sum_{j} F(i, j)$ for all $i$ 's. Now, define the digraph gap $g(G)=\max _{S} \sum_{i \in S}\left|\sum_{j \in \bar{S}}\left(F_{\pi}(i, j)-F_{\pi}(j, i)\right)\right|$, which quantifies the maximum difference between two bipartite subgraphs $S$ and $\bar{S}$ among all partitions. We have the following theorem relating the degree of asymmetry with $g(G)$ and $\sigma_{n-1}(P)$, the second largest singular value of $P$.

Theorem 5 (Bounds on the degree of asymmetry).

$$
2 g(G) \leq \delta_{n} \leq \lambda_{n-1}^{\frac{1}{2}}\left(\tilde{P}^{T} \tilde{P}\right)=\sigma_{n-1}(P),
$$

where $\tilde{P}=\Pi^{\frac{1}{2}} P \Pi^{-\frac{1}{2}}$.

Proof: The proof of this theorem is delegated to the technical report [12].

Theorem 6 below relates and bounds the second smallest singular value $\sigma_{2}$ of $\tilde{\mathcal{L}}$ in terms of the degree of asymmetry $\delta_{n}$, the Cheeger constant, and the second smallest eigenvalue $\bar{\lambda}_{2}$ of $\overline{\mathcal{L}}$. The proof of this theorem is delegated to the technical report [12].

Theorem 6 (Relations among $\sigma_{2}, \bar{\lambda}_{2}, \delta_{n}$ and the Cheeger constant). Given a strongly connected graph $G=(V, E, A)$, and its Laplacian matrix $\tilde{\mathcal{L}}=\Pi^{\frac{1}{2}}(I-P) \Pi^{-\frac{1}{2}}$, we have the bounds for the second smallest singular value of $\tilde{\mathcal{L}}$ as

$$
\frac{h^{2}(G)}{2} \leq \sigma_{2} \leq\left(1+\frac{\delta_{n}}{\bar{\lambda}_{2}}\right) \cdot 2 h(G) .
$$

When the graph is undirected, we have $\frac{h^{2}(G)}{2} \leq \sigma_{2}=\bar{\lambda}_{2} \leq 2 h(G)$, which is exactly the same as the bounds obtained in [6].

Finally, we introduce a generalized Cheeger constant, $\tilde{h}(G)$, defined as

$$
\tilde{h}(G)=\min _{S} \frac{\left\|\tilde{\mathcal{L}} x_{S}\right\|}{\left\|x_{S}\right\|}=\min _{x_{S} \perp \pi^{\frac{1}{2}}} \frac{\left(x_{S}^{T} \tilde{\mathcal{L}}^{T} \tilde{\mathcal{L}} x_{S}\right)^{\frac{1}{2}}}{\left(x_{S}^{T} x_{S}\right)^{\frac{1}{2}}}
$$


where for any $S \subset N:=\{1,2, \ldots, n\}, x_{S}=\Pi^{-\frac{1}{2}} f_{S}$ is defined above. We see that the generalized Cheeger constant thus defined minimizes the 2-norm of the circulations across bipartite subgraphs $S$ and $\bar{S}$, whereas $h(G)$ minimizes the 1-norm (the sum of absolute values) of the circulations across $S$ and $\bar{S}$. Clearly, $\sigma_{2} \leq \tilde{h}(G)$.

\subsection{Bounding the Mixing Rate of Random Walks on Digraphs}

In this section, using mixing rating bounds as an example, we show that by considering the degree of asymmetry, we obtain a better bound for the mixing rate of random walks on digraphs.

The mixing rate is a measure of how fast a random walk converges to its stationary distribution. Many papers have studied the problem of bounding the mixing rate of random walks (or reversible Markov chains) on undirected graphs, such as $[4,11]$.Relatively few papers $[6,9,14]$ have addressed the problem of bounding the mixing rate of Markov chains (or random walks) on digraphs. In bounding the convergence rate from an initial distribution to the stationary distribution of a Markov Chain with the transition probability matrix $P$, the $\chi$-square distance $[6,9]$ is commonly used, and is defined as follows:

$$
\chi(t)=\max _{i \in V(G)}\left(\sum_{j \in V(G)} \frac{\left(P^{t}(i, j)-\pi_{j}\right)^{2}}{\pi_{j}}\right)^{\frac{1}{2}} .
$$

Fill in [9] derives an upper bound on the mixing rate of a random walk on digraphs in terms of the second smallest eigenvalue, $\bar{\lambda}_{2}$ of $\overline{\mathcal{L}}$. When the Markov chain $P$ is strongly aperiodic, define $\tilde{P}=\Pi^{\frac{1}{2}} P \Pi^{-\frac{1}{2}}$, then $\chi^{2}(t) \leq \varepsilon^{t} \max _{i} \pi_{i}^{-1}$, where

$$
\varepsilon=\max _{f \perp \pi^{\frac{1}{2}}} \frac{f^{T} \tilde{P}^{T} \tilde{P} f}{f^{T} f}=\max _{f \perp \pi^{\frac{1}{2}}} \frac{\|\tilde{P} f\|^{2}}{\|f\|^{2}} \leq 1-\bar{\lambda}_{2}
$$

From Theorem 4, we know that this bound leads to the same upper bound for all digraphs with the same $\overline{\mathcal{L}}$. By accounting for the degree of asymmetry, we obtain a lower bound and a (generally) tighter upper bound on $\frac{\|\tilde{P} f\|^{2}}{\|f\|^{2}}$ as follows, which in turn yields a tighter bound on $\chi(t)$ :

Theorem 7. For a strongly aperiodic Markov chain P,

$$
\delta_{n}^{2} \leq \varepsilon=\max _{f \perp \pi^{\frac{1}{2}}} \frac{\|\tilde{P} f\|^{2}}{\|f\|^{2}} \leq\left(1-\bar{\lambda}_{2}\right)^{2}+2 \delta_{n} \bar{\lambda}_{n}+\delta_{n}^{2}
$$

Proof : First, the lower bound can be obtained from Theorem 5. To prove the upper bound, we note that

$\frac{f^{T} \tilde{P}^{T} \tilde{P} f}{f^{T} f}=\frac{f^{T}(\overline{\mathcal{L}}-I)^{2} f}{f^{T} f}+\frac{f^{T}\left(\overline{\mathcal{L}} \Delta+\Delta^{T} \overline{\mathcal{L}}+\Delta^{T} \Delta\right) f}{f^{T} f} \leq\left(1-\bar{\lambda}_{2}\right)^{2}+2 \delta_{n} \bar{\lambda}_{n}+\delta_{n}^{2}$. 
The above theorem states that the mixing rate of a random walk on a digraph cannot be slower than $\delta_{n}^{2}$; and it is upper bounded by a function of $\bar{\lambda}_{2}, \bar{\lambda}_{n}$ and $\delta_{n}$. In particular, when $\tilde{\mathcal{L}}$ is symmetric (i.e., the underlying graph is undirected), the bound in eq.(31) reduces to

$$
\varepsilon=\max _{f \perp \pi^{\frac{1}{2}}} \frac{\|\tilde{\mathcal{P}} f\|^{2}}{\|f\|^{2}} \leq\left(1-\bar{\lambda}_{2}\right)^{2},
$$

where the equality $\varepsilon=\left(1-\bar{\lambda}_{2}\right)^{2}$ is attained. In contrast, eq.(30) yields the bound as $\varepsilon=1-\bar{\lambda}_{2}$. Hence when the underlying graph is undirected, our bound is tighter.

As a final remark, we note that similar derivations can be applied to obtain a tighter bound (than that of Chung's [6]) on the mixing rate of lazy random walk on $G$ with transition probability matrix $\mathcal{P}=\frac{I+P}{2}$ (see [12]).

\section{References}

1. D. Aldous and J. A. Fill. Reversible markov chains and random walks on graphs. http://www.stat.berkeley.edu/ Aldous/RWG/book.html.

2. R. Bhatia. Matrix Analysis. Springer, 1997.

3. D. Boley, G. Ranjan, and Z.-L. Zhang. An asymmetric laplacian for a directed graph. University of minnesota computer science department technical report: Tr09-009, 2009.

4. S. Boyd, A. Ghosh, B. Prabhakar, and D. Shah. Mixing times for random walks on geometric random graphs. In SIAM Workshop on Analytic Algorithmics and Combinatorics (ANALCO), pages 240-249, Vancouver, 2005. SIAM.

5. S. Brin and L. Page. The anatomy of a large-scale hypertextual web search engine. Computer Networks and ISDN Systems, 30(1-7):107-117, 1998.

6. F. R. K. Chung. Laplacians and the cheeger inequality for directed graphs. Annals of Combinatorics, 9:1-19, sep 2005.

7. F. R. K. Chung. Spectral Graph Theory (CBMS Regional Conference Series in Mathematics, No. 92). Cbms Regional Conference Series in Mathematics, 2006.

8. F. R. K. Chung and S. T. Yau. Discrete green's functions. Journal of Combinatorial Theory, Series A, pages 191-214, July 2000.

9. J. A. Fill. Eigenvalue bounds on convergence to stationarity for nonreversible Markov chains, with an application to the exclusion process. Ann. Appl. Probab., 1(1):62-87, 1991.

10. R. Horn and C. R. Johnson. Matrix Analysis. Cambridge University Press, 1st edition, 1985.

11. M. Jerrum and J.-B. Son. Spectral gap and log-sobolev constant for balanced matroids. the 43rd Annual IEEE Symposium on Foundations of Computer Science (FOCS 2002), 2002.

12. Y. Li and Z.-L. Zhang. Random walks on diagraphs, the generalized digraph laplacian and the degree of asymmetry. Technical Report, http://www.cs.umn.edu/ yanhua/, CSE Department of University of Minnesota, 2010.

13. L. Lovàsz. Random walks on graphs: A survey. Combinatorics, 2:1-46, 1993.

14. M. Mihail. Conductance and convergence of markov chains-a combinatorial treatment of expanders. Proceedings of FOCS'1989, 0:526-531, 1989.

15. D. Zhou, J. Huang, and B. Schölkopf. Learning from labeled and unlabeled data on a directed graph. In ICML '05: Proceedings of the 22nd international conference on Machine learning, pages 1036-1043, New York, NY, USA, 2005. ACM. 